\title{
Fast and Room-temperature Synthesis of Porous Alumina Films in Ultrasonic Assisted Bath Inducing Superficial Cavitations
}

\author{
V.I.M. Paulo, ${ }^{a}$ J. Neves-Araujo ${ }^{a}$ and E. Padrón-Hernández ${ }^{a, b, *}$ \\ ${ }^{a}$ Programa de pós-graduação em Ciência de Materiais, Universidade Federal de Pernambuco, \\ Recife, PE 50670-901, Brasil

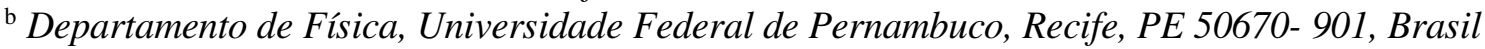

Received December 16, 2016; accepted April 14, 2017

\begin{abstract}
We made a nano-porous alumina membrane by anodization under $40 \mathrm{~V}$ by two steps (10 minutes and 120 minutes) in oxalic acid, followed by chemical treatment in phosphoric acid assisted by an ultrasonic bath. Thus, we achieved a total relatively short time ( $3 \mathrm{~h} 30 \mathrm{~min}$ ) for the synthesis, having all physicochemical processes been done at room temperature $\left(25^{\circ} \mathrm{C}\right)$. We verified a hexagonal arrangement structure with $56 \mathrm{~nm}$ inter-pore diameter, and density of $10^{10}$ pores $/ \mathrm{cm}^{2}$, characteristic of the self-ordered porous alumina. Therefore, our membranes can be used as a template for fast synthesis of nanostructures as nanowires, nano-tubes.
\end{abstract}

Keywords: Porous alumina; rapid synthesis; room temperature process; ultrasonic bath.

\section{Introduction}

In the last two decades, the structural control of porous anodic alumina (PAA) has been widely investigated due to its use as a template, electronic devices, fotoluminescent, magnetic systems, biotechnology, and others [1]. The structure consists of an alumina membrane $\left(\mathrm{Al}_{2} \mathrm{O}_{3}\right)$ containing pores distributed in numerous hexagonal cells. A PAA membrane may contain a high density of pores $\left(10^{9}-10^{11}\right.$ pores $\left./ \mathrm{cm}^{2}\right)$ so as to have hundreds of pores in a few of square millimeters [2, 3]. PAA membrane can be obtained by electrochemical anodization of pure aluminum foils in and acidic electrolyte. The nano-structural characteristics are determined by the physicochemical factors governing the selfordering process such as the electrolyte type, $\mathrm{pH}$, the applied electric potential, anodization time, temperature, etc. [4-7]. In 1995, Masuda et al. introduced the anodizing method through two steps [8]. They managed an improvement in the degree of ordering for the PAA, which increased its importance from the point of view of nanofabrication. The specific conditions for self-ordering of PAA nanopores for various types of electrolytes were studied. The fabrication is typically

\footnotetext{
* Corresponding author. E-mail address: padron@df.ufpe.br
} 
characterized by a long period of anodization under a constant potential specific for each electrolyte type [7]. The ordering in a long time (160 hours) occurs under $40 \mathrm{~V}$ for an oxalic acid $\left(\mathrm{H}_{2} \mathrm{C}_{2} \mathrm{O}_{4}\right)$ solution, $25-27 \mathrm{~V}$ in sulfuric acid $\left(\mathrm{H}_{2} \mathrm{SO}_{4}\right)$ solution and $195 \mathrm{~V}$ for phosphoric acid $\left(\mathrm{H}_{3} \mathrm{PO}_{4}\right)$ solution, all at $0{ }^{\circ} \mathrm{C}$ [8 10]. The three solution types have been frequently used as anodizing electrolytes. However, the long time for anodizing is the factor determining the degree of ordering for the pores in membrane $[7,8,11]$. On the other hand, the membrane structure can be modified by post-anodizing procedures. This involves the etching for cleaning and opening the pores with the immersion of the sample in a corrosion solution. Usually is used a phosphoric acid $\left(\mathrm{H}_{3} \mathrm{PO}_{4}\right)$ solution or a mixture of phosphoric and chromic acids $\left(\mathrm{H}_{3} \mathrm{PO}_{4}+\mathrm{H}_{2} \mathrm{CrO}_{4}\right)$ for the corrosion step ranging from 30 up to $60{ }^{\circ} \mathrm{C}$ [11-13]. Thus, the chemical etching step, if used appropriately, can improve the morphology of the PAA structure and enables the reduction of the synthesis time. In this scenario, the reduction of the time to obtain the porous membrane at room temperature is an important point in the academic and industrial field because it can improve search/applicability of the PAA and the cost-benefit ratio.

In the present study, we prepared a self-ordered porous alumina formed by anodization in $0.3 \mathrm{M}$ aqueous solution of $\mathrm{H}_{2} \mathrm{C}_{2} \mathrm{O}_{4}$ in potentiostatic regime under $40 \mathrm{~V}$. All steps of the synthesis process were at room temperature of $25^{\circ} \mathrm{C}$ and the total process time was relatively short, about $3 \mathrm{~h} 30 \mathrm{~min}$. For this proposal we made some adjustments to the experimental procedure of anodizing by two steps [8]. The main modification was in the chemical treatment with $\mathrm{H}_{3} \mathrm{PO}_{4}$ solution using an ultrasonic bath after the first and second anodization. The production by using the ultrasound process is based on the creation of implosions conducing to the formation of cavities with steam and gas (called cavitations) in a liquid. This promotes activation effects in chemical reactions at high temperature of about $5000{ }^{\circ} \mathrm{C}$ and pressure of about 500 atm $[14,15]$. These conditions are favorable for accelerating the chemical reactions for the self-organizing processes. With the introduction of an ultrasonic bath we contributed to the material removal and then the formation of PAA membranes with self-ordered hexagonal arrangement.

\section{Experimental}

Alumina membranes were prepared by anodization process in two steps using a potentiostat (IviumStat) and an ultrasonic washer (Fisher Scientific -FS14) operating at $25 \mathrm{kHz}$. Aluminum sheets with $0.5 \mathrm{~mm}$ of thickness $(99.999 \%$ purity) and area of $1.5 \mathrm{~cm}^{2}$ were introduced in a $40 \mathrm{~mL}$ glass container used as the electrolytic cell for anodization and a rectangular piece of graphite was used as the cathode. The sheet was washed with acetone for 10 minutes for the surface cleaning, then it was electro-polished under current density of $330 \mathrm{~A} / \mathrm{m}^{2}$ in a $\mathrm{H}_{2} \mathrm{SO}_{4}+\mathrm{H}_{2} \mathrm{O}+\mathrm{H}_{3} \mathrm{PO}_{4}$ (ratio 1: 2: 2 in volume) solution for 10 min to decrease the surface roughness. Then, a first anodization took place in a solution of oxalic acid $0.3 \mathrm{M}$ for 10 min under constant voltage of $40 \mathrm{~V}$. A chemical etching in 1.0 $M$ solution of phosphoric acid, assisted by an ultrasonic bath for $36 \mathrm{~min}$, was performed. The sheet was re-anodized in the same conditions of the first step by 
120 minutes. A subsequent 7 minutes, ultrasonic assisted, etching was performed under the same conditions mentioned above. Finally the sheet was washed in deionized water for $15 \mathrm{~min}$, in ultrasonic bath again. All procedures were performed at room temperature $\left(25^{\circ} \mathrm{C}\right)$. The characterization of the specimen was examined by a scanning electron microscope (JEOL JSM-5900 and FEI Quanta 200F) and the subsequent image processing by the open access software ImageJ [16].

\section{Results and discussions}

Hexagonal array of nanopores can be obtained by anodizing the aluminum, if applied a constant electric potential (potentiostatic regime) or a constant current (galvanostatic regime). According to the model of anodic porous alumina growth, the current-time transient obtained by anodizing at constant voltage results in the growth of two alumina film, a barrier layer and one porous layer $[17,18]$. Fig. 1 shows the change in current density during the first and second anodization at $40 \mathrm{~V}$. The growth kinetics for the PAA in potentiostatic regime includes steps detectable by monitoring the dependence of the current density in the anodization time. We observed that the current density suffers a fast fall with time in the two-step anodizing until it reaches a minimum. Then gradually increases to a maximum and then decreases linearly (Fig. 1).

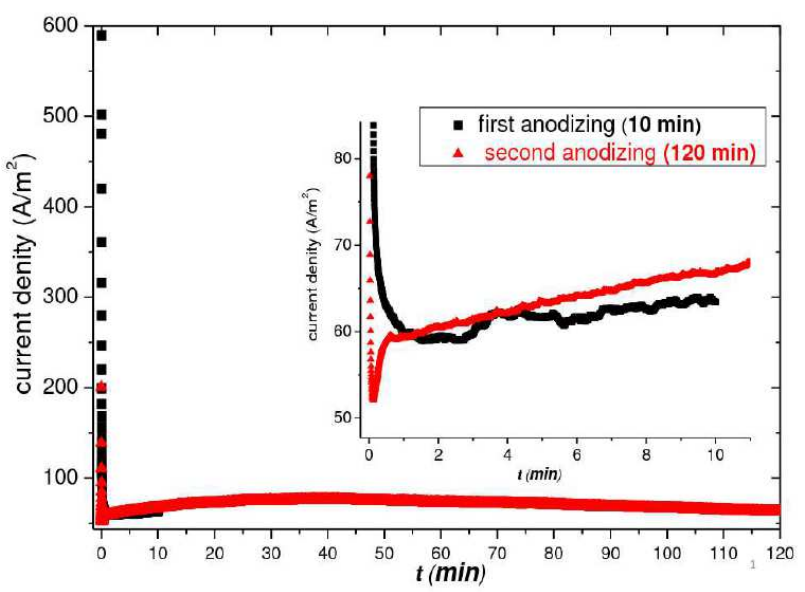

Figure 1. Current versus time curves for the first and second anodization (10 and 120 $\min )$ at $25^{\circ} \mathrm{C}$ in oxalic acid.

The initial stage (up to the minimum) corresponds to the time interval for the growth of non-porous barrier layer. That range of about $2.6 \mathrm{~min}$ in the first anodization and $0.1 \mathrm{~min}$ in the second, is characterized by increasing the current density. After this stage it occurs the growth of the porous layer. The periodic features formed on the aluminum surface during the first anodizing serve as nucleation sites for the formation of the nanopores in the second anodizing $[2,3]$. We will see later that the chemical treatment in $\mathrm{H}_{3} \mathrm{PO}_{4}$ solution assisted by ultrasonic bath was a key step after the first and second anodization for the pores formation. Through scanning microscopy we verified the morphology of the structure in different conditions of post-anodization chemical process. We fixed 
the time for the first and second anodizing in $10 \mathrm{~min}$ and $120 \mathrm{~min}$, respectively. Then the samples prepared with chemical treatment in $\mathrm{H}_{3} \mathrm{PO}_{4}$ without/under ultrasonic assisted bath were analysed to assess the topological and structural changes in the formed membrane.
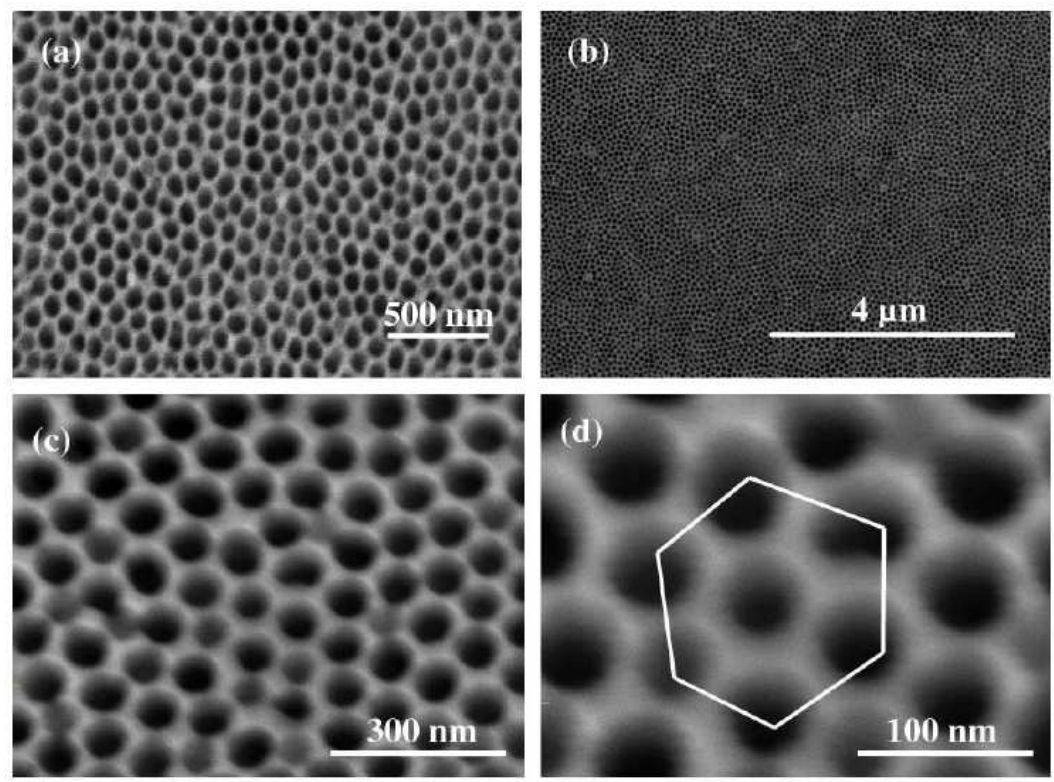

Figure 2. SEM images of the surface view of the membrane. (a) PAA prepared without ultrasonic assisted bath, and (b-d) prepared with ultrasonic assisted bath. Magnification of 280k x and 600k $x$ in (c) and (d), respectively. Inset hexagonal array (d).

Samples prepared without ultrasonic assisted bath at $25{ }^{\circ} \mathrm{C}$, verified by SEM (Fig.2a), do not present a formation of pores organization uniformly on the membrane surface. We can see irregular pores not open completely, but with a hexagonal spatial distribution. This demonstrates that the anodization followed by chemical treatment without $\mathrm{H}_{3} \mathrm{PO}_{4}$ was not sufficient for efficient formation of the porous membrane. So in a second step we use the ultrasonic assisted bath in an attempt to improve the morphology of the structure. Therefore, we remade the corrosion process with $\mathrm{H}_{3} \mathrm{PO}_{4}$ under the same conditions of the first step, but now on the ultrasonic assisted bath post-anodization.

This modification was sufficient to improve the structure of the membrane and reduce the process time for 3 hours and $30 \mathrm{~min}$. The prepared samples have porous surface morphology, as shown in Fig. 2 (b-d). In Fig. $2 b$ there is a porous array for long range, on the membrane surface. Fig. $2 \mathrm{c}-\mathrm{d}$ show magnifications of $280 \mathrm{k} x$ and $600 \mathrm{k} x$ respectively verifying the pores formation around $50-60 \mathrm{~nm}$ and distributed periodically. We identified the array of nano-pores in a hexagonal spatial distribution in Fig. 2d, characteristic of self-arrangement of the PAA [3].

The start point of the self-ordering process occurs with aluminum subjected to the first anodizing step. The concave periodicals geometries are formed on the aluminum surface in this step and serve as nucleation sites for the formation of pores in the second anodizing [3]. We suggest that the chemical treatment process in $\mathrm{H}_{3} \mathrm{PO}_{4}$ under ultrasonic assisted bath not only cleans the surface by removing the non-porous oxide, but also promotes nucleation sites for growth of 
nanopores. This leads to the improvement in the porous surface of the PAA as verified by SEM micrographs. On the other hand, it is important to mention the features obtained for the walls of the nanopores, as well as the thickness of the layer of porous PAA. These can be modified by post-treatment procedures involving a chemical etching. We analyzed the cross section of the membrane prepared in ultrasonic assisted bath (Fig. 3).
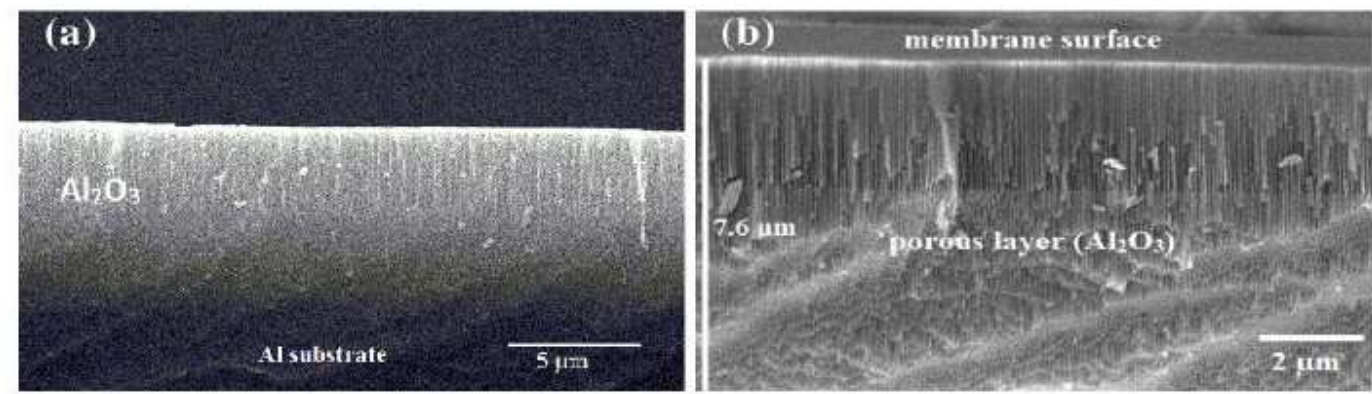

Figure 3. Image of the cross section for the membrane. (a) image with regions of the alumina substrate and Al. (b) Profile of the porous membrane layer.

Fig. 3a shows a lateral profile of membrane with a porous layer of $\mathrm{Al}_{2} \mathrm{O}_{3}$ formed on the anodized aluminum substrate. A porous film of more than $7 \mu \mathrm{m}$ thick was formed on the Al surface, as shown in Fig. 3b. It is possible to verify that channels of the pores are perpendicular to the membrane surface. Thus we see that our structure has the typical characteristics of PAA self-ordered [3]. Therefore, we conclude that the ultrasonic assisted bath was fundamental in the obtaining of PAA structure. Applied the ultrasonic assisted bath, cavitations phenomenon occurs with the implosion of $\mathrm{H}_{3} \mathrm{PO}_{4}$ micro-bubble, generating cavities in aluminum oxide particles. W. J. Tonilson et al. reported the cavitation process for alumina in distilled water by ultrasound and suggested that cavitation bubbles preferentially attack the grain boundaries of the particles oxide [17]. The same process made in a corrosive liquid such as $\mathrm{H}_{3} \mathrm{PO}_{4}$ solution is more intense.
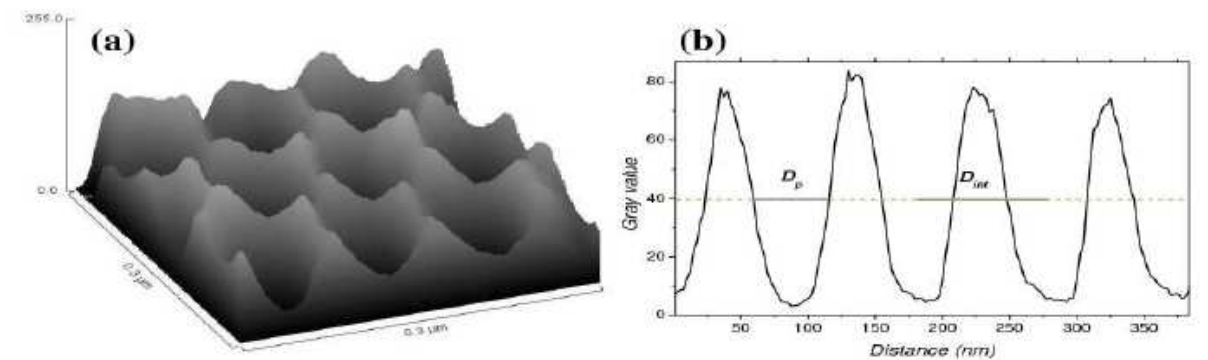

Figure 4. Charts obtained with ImageJ. (a) Gray contrast to the selected area of the region of Fig. 2 (d). (b) A pore distribution along a pores line in Fig. 2 (d).

According to Suslick et al., $\mathrm{H}_{3} \mathrm{PO}_{4}$ with low volatility and high solubility makes more efficient collapse of bubbles [18]. Consequently, the cavity in the oxide particles increases in size, while remaining a core, favoring the nucleation points for formation of pores arranged in the second anodizing. Thus, if performed in the last chemical treatment under ultrasonic assisted bath, the structure is obtained with the geometry features of self-ordering as observed by SEM in Fig. 
2 (b-d). To explore the geometric characteristics of PAA structure we used software ImageJ for image processing [16]. ImageJ is an image processor that allows a qualitative and quantitative analysis using the micrograph images. We have taken the region of Fig. 2 d, and plotted the superficial gray contrast shown in Fig. 4a.

The contrast shows that the membrane surface topography is relatively well distributed in a hexagonal array of nanopores. To measure the distance between the pores and the size of the porous cell we plotted contrast profile along a line of pores of Fig. 2d, as shown in Fig. 4b. From this graph it was measured the average period of the function, which coincides with the inter-pore distance $\left(D_{\text {int }}\right)$. Average distance between two valleys in the graph corresponds to $D_{\text {int }}=$ $94 \mathrm{~nm}$. To get the pore diameter $\left(\mathrm{D}_{\mathrm{p}}\right)$ we calculated the average distance between two maxima at half-height, finding $D_{p}=56 \mathrm{~nm}$ and the nanopores radius $r=28$ $\mathrm{nm}$. Now we can find the superficial density of pores $(\sigma)$ and the porosity of the membrane or packing factor $(\alpha)$. Density may be calculated by the following formula [7]: $\sigma=\left(2 \times 10^{14} / \sqrt{ } 3\right) \times D_{\text {int }}$. Substituting $D_{\text {int }}$ by $94 \mathrm{~nm}$, we obtain $1.3 \times 10^{10}$ pores $/ \mathrm{cm}^{2}$, density value in the range reported for self-ordered PAA [2, 3]. Nielsch et al. described, formed PAA by the aluminum anodizing and selforganizing conditions and suggested that porosity can be calculated from equation $\alpha=2 \pi / \sqrt{ } 3 \times\left(r / D_{\text {int }}\right)^{2}$ [19]. Substituting the values of $r=28 \mathrm{~nm}$ and $D_{\text {int }}$ $=94 \mathrm{~nm}$ we obtain $\alpha=32 \%$. This value is greater than the $10 \%$ obtained in the mentioned paper because chemical attack in $\mathrm{H}_{3} \mathrm{PO}_{4}$ enlarges the diameter of the pores in the synthesis process [19]. However, we obtained in a fast procedure the structure of self-ordained with pores arranged in a hexagonal configuration.

\section{Conclusion}

In summary, we synthesized a self-ordered porous alumina in a few hours of processing ( 3 hours and 30 minutes) conducted at $25^{\circ} \mathrm{C}$. The process consists in using a $\mathrm{H}_{3} \mathrm{PO}_{4}$ chemical etching under ultrasonic assisted bath post-anodizing. The cavitations process generated in the under ultrasonic assisted bath not only cleans the membranes but also improves the process of nucleation and formation of the pores. We verified by the treatment of the SEM image that our porous alumina has the structural features of self-arrangement reported in the literature. Therefore, this porous alumina membrane can be used as a template or mask for manufacturing nanostructured systems.

\section{Acknowledgements}

This work has been supported by the Brazilian Agencies CNPq, FINEP, CAPES and FACEPE.

\section{References}

1. Poinern GEJ, Ali N, Fawcett D. Materials. 2011;4:487-526.

2. Li AP, Müller F, Birner A, et al. J Appl Phys. 1998;84:6023-6026. 
3. Lee W, Park SJ. Chem Rev. 2014;114:7487-7556.

4. Ono S, Saito M, Asoh H. Electrochim Acta. 2005;51:827-833.

5. Araoyinbo AO, Rahmat A, Derman MN, et al. Adv Mat Lett. 2012;3:273278.

6. $\quad$ Ba L, Li WS. J Phys D: Appl Phys. 2000;33:2527.

7. Lee W, Ji R, Gosele U, et al. Nature Mater. 2006;5:741-747.

8. Masuda H, Fukuda K. Science. 1995;268:1466-1468.

9. Masuda H, Hasegwa F, Ono S. J Electrochem Soc. 1997;144:L127-L130.

10. Masuda H, Yada K, Osaka A. Jap J Appl Phys. 1998;37:L1340.

11. Stępniowski WJ, Nowak-Stępniowska A, Presz A, et al. Mater Charact. 2014;91:1-9.

12. Li AP, Müller F, Birner A, et al. J Vacuum Sci Technol A. 1999;17:14281431.

13. Xu JP, Zhang ZZ, Ma B, et al. J Appl Phys. 2011;109:07B704.

14. Lindley J, Mason T. Chem Soc Rev. 1987;16:275-311.

15. Rooze J, Rebrov EV, Schouten JC, et al. Ultrasonics Sonochem. 2013:20:111.

16. ImageJ, National Institute of Mental Health, Bethesda, MD, USA.http://rsb.info.nih.gov/ij

17. Tomlinson WJ, Kalitsounakis N, Vekinis G. Ceramics Int. 1999;25:331338.

18. Suslick KS, Eddingsaas NC, Flannigan DJ, et al. Ultrasonics Sonochem. 2011;18:842-846.

19. Nielsch K, Choi J, Schwirn K, et al. Nano Lett. 2002;2:677-680. 